\title{
Cationic liposome-mediated nitric oxide synthase gene therapy enhances the antitumor effects of cisplatin in lung cancer
}

\author{
SUJUAN YE*, WEIHAN YANG ${ }^{*}$, YU WANG, WENJING OU, QINGPING MA, CHUANJIANG YU, \\ JIANG REN, GUOXING ZHONG, HUASHAN SHI, ZHU YUAN, XIAOLAN SU and WEN ZHU \\ State Key Laboratory of Biotherapy and Cancer Center, West China Hospital, \\ Sichuan University, Chengdu, Sichuan 610041, P.R. China
}

Received June 16, 2012; Accepted August 2, 2012

DOI: $10.3892 /$ ijmm.2012.1171

\begin{abstract}
Cisplatin is one of the most effective antitumor drugs for non-small cell lung carcinoma (NSCLC) patients. However, its efficacy has encountered a plateau due to its side effects and drug resistance. Inducible nitric oxide (NO) synthase (iNOS) gene therapy has been reported to have antitumor effects in several types of cancers and enhances sensitivity to cisplatin, but the effects of $i N O S$ gene therapy alone or its combination with cisplatin in lung cancer remain unclear. In the current study, we evaluated the effects of cationic liposome (LP)-mediated iNOS gene transfection on enhancing low-dose cisplatin-mediated antitumor effects in the A549 human lung adenocarcinoma cell line in vitro. Furthermore, we examined whether iNOS gene therapy enhances the antitumor effects of low-dose cisplatin in two A549 human lung cancer cell xenograft mouse models. The results revealed that $i N O S$ gene therapy may significantly enhance low-dose cisplatin-mediated inhibition of cell proliferation, invasion, migration and promotion of cell apoptosis in A549 cells. Intratumoral administration of the LP-pVAX-iNOS complex significantly enhanced lowdose cisplatin-mediated suppression of subcutaneous tumor growth. Moreover, intravenous injection of the LP-pVAXiNOS complex greatly enhanced low-dose cisplatin-mediated inhibition of experimental lung metastasis and prolonged the life span of mice without significant organ-related toxicity in a nude mouse model of lung metastasis compared to the cisplatin alone-treated group. Furthermore, iNOS gene-mediated
\end{abstract}

Correspondence to: Professor Wen Zhu, State Key Laboratory of Biotherapy and Cancer Center, West China Hospital, Sichuan University, No. 1 Keyuan 4th Road, Gaopeng Street, High Technological Development Zone, Chengdu, Sichuan 610041, P.R. China

E-mail: zwjulia@163.com

*Contributed equally

Key words: iNOS, cisplatin, A549, lung cancer, cationic liposomes, gene therapy, combination treatment enhancement of cisplatin-mediated antitumor effects in lung cancer may be related to the attenuation of p-mTOR, MMP2 and the activation of p-p53. Thus, the combination treatment with $i N O S$ gene therapy and cisplatin may be a novel and effective therapeutic strategy for lung cancer.

\section{Introduction}

Lung cancer is the leading cause of cancer-related mortality worldwide. Among all types of lung cancers, non-small cell lung carcinoma (NSCLC) accounts for approximately $85 \%$ of all lung cancer cases (1). As the first-line chemotherapeutic agent in lung cancer, cisplatin, has encountered a plateau due to its side effects and lack of specificity $(2,3)$. Therefore, it is particularly urgent to discover methods by which to enhance the chemosensitivity of cisplatin.

Inducible nitric oxide (NO) synthase (iNOS) is a new promising cancer target gene involved in NO-mediated antitumor effects (4-7). It was first identified and characterized in cytokine-activated murine macrophages (5). The relatively lower expression of the $i N O S$ gene was observed in several types of human cancers, where it is difficult to maintain high levels of NO for long periods of time $(6,8,9)$, while full expression of the iNOS gene may generate high concentrations of NO for prolonged periods of time (10). Generally, cytokine stimulation, NO donor and iNOS gene transfer have been identified as the main methods used to upregulate the expression of $i N O S$ and further produce high concentrations of NO in human cancer cells (4). Among all known methods, however, cytokine stimulation and NO donor may cause hypotension, drug resistant, toxic and other side effects, while iNOS gene transfer may essentially avoid the above side effects $(11,12)$. iNOS gene transfer has a unique feature of a bystander effect, which would be an absolute requirement to the future success of cancer gene therapy as a contribution to the high efficiency of gene transfer $(13,14)$.

The forced high level of NO was reported to have antitumor activities (15). In addition, clinical studies have demonstrated that overexpression of the $i N O S$ gene may increase the survival of colorectal, ovarian and NSCLC patients $(9,16,17)$. The apparent antitumor effects of a high NO level generated by $i N O S$ gene transfer have been confirmed in 
several types of cancers including breast, colorectal, prostate, ovarian, melanoma, kidney, thyroid cancer in vitro and in vivo (7,18-24). Thus, high concentrations of NO generated from the full expression of the $i N O S$ gene may play an important role in cancer treatment.

Notably, studies have also demonstrated that high concentrations of NO generated from iNOS gene transfer, cytokine stimulation or NO donor may enhance the cytotoxicity of the chemotherapeutic drug cisplatin in RIF-1 tumors, ovarian cancer, leukemia, prostate cancer, colon cancer cells and lung fibroblasts $(18,25-27)$. It is proposed that NO enhances cisplatin toxicity through inhibition of the repair enzymes that act on cisplatin-induced DNA damage (18), but the exact mechanisms of the enhanced effects of iNOS gene therapy and cisplatin in human cancers remain unclear. The antitumor effects of high levels of NO generated from $i N O S$ gene transfer combined with cisplatin in lung cancer cells have yet to be reported.

Previous studies have demonstrated that the activity of the iNOS gene was observed in lung adenocarcinoma compared with normal tissues $(28,29)$ and the $i N O S$ gene expression levels are moderate in NSCLC cells as well as in other cancer cells (9). As the stages developed, the expression of $i N O S$ protein gradually decreased in NSCLC cells. The intense expression of NOSs including the $i N O S$ gene seems to be a favorable prognostic sign in NSCLC patients. Based on this, we hypothesize that delivering a high level of the exogenous $i N O S$ gene by gene therapy in lung cancer may generate a large amount of NO and thus enhance the effects of cisplatin in lung cancer treatment.

To identify the hypothesis, in the current study, we first evaluated the effects of cationic liposome (LP)-mediated iNOS gene transfection on enhancing low-dose cisplatin-mediated antitumor effects in the human lung adenocarcinoma A549 cell line in vitro. Based on the in vitro results, we then aimed to demonstrate that intratumoral delivery of the LP-pVAX-iNOS complex enhanced low-dose cisplatin-mediated suppression of tumor growth. Meanwhile, systemic delivery of the LP-pVAXiNOS complex enhanced low-dose cisplatin-mediated inhibition of experimental lung metastasis and prolonged animal survival. Apoptosis was further detected in the subcutaneous tumor tissues. Furthermore, we primarily detected several cellular targets involved in NO- and/or cisplatin-mediated signaling pathways during the antitumor procedure to understand the molecular mechanism of the enhanced effects by western blotting. This study may provide a new method for clinically effective treatment of lung cancer.

\section{Materials and methods}

Cell cultures, chemotherapeutic drug and animals. Human lung adenocarcinoma cell line A549 was obtained from the American Type Culture Collection (ATCC, Manassas, VA, USA) and was grown in RPMI-1640 medium (Gibco-BRL, Grand Island, NY, USA) supplemented with $10 \%$ fetal bovine serum $(\mathrm{FBS})$ at $37^{\circ} \mathrm{C}$ in a humidified $5 \% \mathrm{CO}_{2}$ atmosphere. Cisplatin was obtained from the West China Hospital pharmacy. Female athymic BALB/c nude mice were purchased from the Shanghai Laboratory Animal Centre (SLAC, Shanghai, China). The mice were housed in laminar flow cabinets under specific pathogen-free conditions. All animal experiments were performed in accordance with the institutional guidelines established for animal care and use.

Construction of iNOS gene expression vector and preparation. According to the $i N O S$ cDNA coding sequence (GeneBank serial no., BC130283.1), the CDS sequence of the $i N O S$ gene was cloned from $i N O S$-pCR4-TOPO plasmid (Open Biosystems, USA) with a PrimeSTAR ${ }^{\mathrm{TM}}$ HS PCR kit (Takara, Dalian, China) and connected to the pVAX plasmid vector (Invitrogen Life Technologies, Carlsbad, CA, USA). Pure pVAX and pVAX-iNOS plasmids were prepared using an EndoFree ${ }^{\mathrm{TM}}$ Plasmid Giga kit (Qiagen, Chatsworth, CA, USA), measured for concentration using a spectrophotometer and diluted to $1 \mu \mathrm{g} / \mu \mathrm{l}$ of DNA.

Preparation of plasmid LP-DNA complexes for cell transfection and animal treatment. Preparations of plasmid LP-DNA complexes were previously described (30). Briefly, $\mathrm{pVAX} / \mathrm{pVAX}-i N O S$ plasmid and LPs diluted in equal volumes of RPMI-1640 were mixed to form LP-DNA complexes (LP-pVAX and LP-pVAX-iNOS) according to their molecular weight ratio (1:6). Cells were transfected with the complexes for $4 \mathrm{~h}$ and replenished with RPMI-1640 medium supplemented with $10 \%$ FBS and $i N O S$ gene co-factor tetrahydrobiopterin $\left(\mathrm{BH}_{4}\right)$ (Sigma, St. Louis, MO, USA) $\left(1 \times 10^{-5} \mathrm{M}\right)$ if cells were transfected with LP-pVAX-iNOS and incubated for another $48 \mathrm{~h}(18,19,21,22)$. The transfection efficiency was evaluated by a parallel transfection with an equal amount of enhanced green fluorescent protein-expressing plasmid vector $\mathrm{p}$-EGFP-N1 (Clontech, Beijing, China) in each of the cell lines.

For the animal experiments, pVAX/pVAX-iNOS plasmid and LPs diluted in equal volume of $5 \%$ glucose were mixed to form a final concentration of $20 \mu \mathrm{g}$ DNA-4 mM LPs in $200 \mu \mathrm{l}$ final volume (weight ratio 1:7). Particle size and $\zeta$-potential of plasmid LP-DNA complexes were measured by a Zeta Nano series (Malvern Instruments, Herrenberg, Germany) at room temperature. The average particle size of the complexes was limited from 200 to $350 \mathrm{~nm}$ and the average $\zeta$-potential of the complexes was limited from 20 to $35 \mathrm{mV}$.

Cell viability assay. To assess the sensitivity of the A549 cells to cisplatin, the 3-(4,5)-dimethylthiahiazo(-z-y1)-3,5di-phenytetrazoliumromide (MTT) (Sigma) assay was used. Cells grown in 96-well plates were transfected with LP-DNA complex using the method described above. The transfection media were replaced with fresh medium containing cisplatin with serial concentrations $(0.0-15.0 \mu \mathrm{M})$ and incubated for another $48 \mathrm{~h}$. Twenty microliters of MTT in each well together with $180 \mu \mathrm{l}$ RPMI-1640 were added and incubated for another $4 \mathrm{~h}$. Absorbance at $490 \mathrm{~nm}$ was measured with a microplate reader (Bio-Rad, Hercules, CA, USA). Both the $\mathrm{IC}_{50}$ and $\mathrm{IC}_{20}$ values of cisplatin were calculated using the Origin 8 software (OriginLab Corporation, Northampton, MA, USA; www.OriginLab.com). To detect the inhibition of A549 cell proliferation in the different treatment groups, cells seeded in 96-well plates were treated with LPs-DNA and/or an $\mathrm{IC}_{20}$ dose level of cisplatin. The cell viabilities after a 48-h treatment were quantified by MTT assay as described above. $\mathrm{IC}_{20}$ dose level of cisplatin was used in all of the following in vitro experiments. 
Cell apoptosis assay. To determine the apoptosis rates of A549 cells following different treatments, Hoechst 33258 staining and an Annexin V-FITC Apoptosis Detection kit (KeyGEN, Nanjing, China) and flow cytometry [fluorescence-activated cell sorting (FACS)] were used according to the manufacturer's instructions. A549 cells following different treatments were stained with Hoechst 33258 as previously described (31). Additionally, cells with the same treatments were rinsed with pre-chilled PBS 3 times, trypsinized with EDTA-free trypsin and then rinsed with PBS supplemented with 2\% BSA. Cells were then labeled by Annexin V-PI reagent and analyzed with the aid of FACS for cell apoptosis analysis.

Cell invasion and migration assays. Evaluation of the invasion and migration abilities was performed using cell invasion and migration assays. After treatment with LPs-DNA and/or low-dose cisplatin for $24 \mathrm{~h}$, cells were harvested and $4 \times 10^{4}$ cells of each treatment in RPMI plus $1 \%$ FBS were replaced in the upper chamber. To assess cell invasion, membranes of the Boyden cell system were coated with BD Matrigel ${ }^{\mathrm{TM}}$ (all were from BD Biosciences, Franklin Lakes, NJ, USA). The bottom chamber was filled with RPMI containing 10\% FBS as a chemoattractant. After being incubated for another $24 \mathrm{~h}$, the attached cells in the lower section of the chamber were stained with $0.1 \%$ crystal violet solution (Sigma). The number of invading cells was manually counted as the sum of 3 randomly selected fields at a $\times 20$ magnification. The same experimental design was used for the migration experiments except that the membranes were not coated with BD Matrigel; RPMI plus $1 \%$ FBS were placed in the lower chamber of Millicell systems (Millipore, Billerica, MA, USA).

\section{Animal studies}

In vivo lung metastasis nude mouse model. Lung metastatic tumors were established via tail vein injection of $2 \times 10^{6}$ A549 cells in a volume of $200 \mu 1$ of RPMI-1640 into female BALB/c athymic nude mice (3-4 weeks old). On Day 12 after cell injection, animals were randomly divided into 6 treatment groups (5 mice/group) including glucose, LP-pVAX, cisplatin, LP-pVAX plus cisplatin, LP-pVAX-iNOS and LP-pVAX-iNOS plus cisplatin groups. Gene therapy was administered through tail vein injection at a dose of $20 \mu \mathrm{g}$ /mouse of the LP-DNA complexes. Twenty-four hours later and immediately prior to i.p. injection of low-dose cisplatin ( $2 \mathrm{mg} / \mathrm{kg} / \mathrm{mouse})$, all mice (including the controls) were injected i.p. with $200 \mu \mathrm{l}$ of $10^{-3} \mathrm{M} \mathrm{BH}_{4}$ as previously described $(18,19,21,22)$. Mice were treated every 3 days and a total of 4 injections were administered to all mice. On the 14th day after the last treatment, all mice were anesthetized and their lungs were filled with India ink to count the number of metastases. In addition, the same lung metastasis mouse model (5 mice/group) was established to analyze the effect of the combination treatment on mice survival. After the same administration, mice were fed until all mice in the glucose group were sacrificed. Mice survival curves were assessed according to the Kaplan-Meier method.

In vivo subcutaneous tumor nude mouse model. A subcutaneous tumor nude mouse model was obtained by intradermal injection with $5 \times 10^{6}$ A549 cells in a volume of $100 \mu \mathrm{l}$ of RPMI1640 in the right flank of female BALB/c athymic nude mice
(5-6 weeks old) as previously described (32). When the tumor volume reached $\sim 80 \mathrm{~mm}^{3}$, the same administration procedures for 6 treated groups ( 5 mice/group) as described above were used in this experiment except that mice were treated by the administration of intratumoral injection at a dose of $20 \mu \mathrm{g} / \mathrm{mouse}$ of the LPs-DNA. Tumor size was measured with callipers in 3 dimensions twice every week. The tumor volume was calculated using the following formula: Volume $\left(\mathrm{mm}^{3}\right)$ $=0.52 \times$ length $(\mathrm{mm}) \mathrm{x}$ width $(\mathrm{mm}) \mathrm{x}$ width $(\mathrm{mm})$. When the average tumor volume reached $\sim 1,000 \mathrm{~mm}^{3}$ or the tumors were necrotic, all mice were sacrificed and the tumors were peeled off and fixed in formalin. Growth curves were plotted for each group. Efficiency of the combination treatment was also assessed by the time required for tumors to reach 3 times their volume from the commencement of treatment.

Analysis of subcutaneous tumor cell apoptosis and histology. To evaluate apoptosis, the fixed subcutaneous tumors were evaluated by in situ TUNEL (terminal deoxynucleotidyl transferase-mediated deoxyuridine triphosphate nick end labeling) staining using an In Situ Cell Death Detection kit (Roche Molecular Biochemicals, Indianapolis, IN, USA) according to the manufacturer's instructions (33). The number of apoptotic bodies was counted from tumor tissues at x200 magnification in 20 randomly selected fields. The hematoxylin and eosin $(\mathrm{H} \& \mathrm{E})$ double staining was applied to detect the histology of the tumor tissue and organic tissue paraffin sections as previously described (34).

Western blot analysis. Lung cancer cells following different treatments were collected and lysed in RIPA buffer supplemented with Protease Inhibitor Cocktail Set I (Merck KGaA, Germany). Equal amounts of lysate proteins were separated by electrophoresis on $10 \%$ SDS-polyacrylamide gels, electrotransferred onto PVDF membranes (Millipore) and probed with anti-iNOS, anti-MMP2 (Abcam, Cambridge, MA, USA), anti-phosho-p53, anti-p53, anti-phospho-mTOR, anti-mTOR (Cell Signaling Technology, Inc., Beverly, MA, USA) and anti$\beta$-actin (Santa Cruz Biotechnology, Santa Cruz, CA, USA). Protein bands were detected using an enhanced ECL system (GE Healthcare Life Sciences, Piscataway, NJ, USA).

Statistic analysis. SPSS13.0 software was used. Each experiment was performed at least 3 times. The data are expressed as the means \pm SD and one-way ANOVA and an unpaired Student's t-test were used to determine the significant differences of all the results. $\mathrm{P}<0.05$ was considered to indicate a statistically significant difference.

\section{Results}

High expression level of iNOS protein on the sensitivity of A549 cells to cisplatin. In this study, the expression of $i N O S$ protein in the A549 cell line after LP-pVAX-iNOS transfection was first detected by western blotting. The moderate expression level of $i N O S$ protein was demonstrated in A549 cells, while LP-pVAX-iNOS transfection induced high expression of the $i N O S$ protein in A549 cells (Fig. 1A). To evaluate whether enforced high expression of the $i N O S$ gene sensitizes the response of A549 cells to 
A

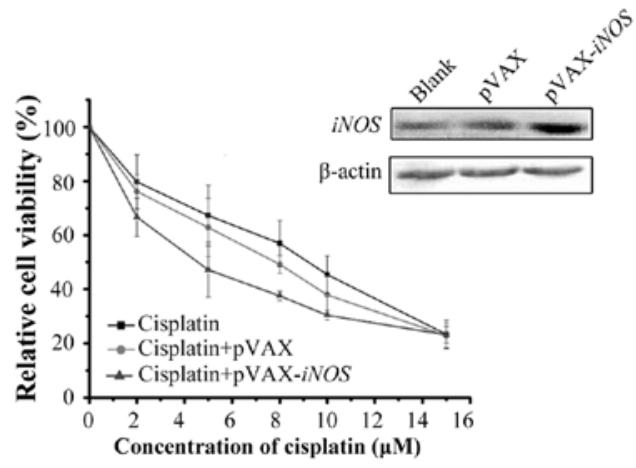

$\mathrm{C}$
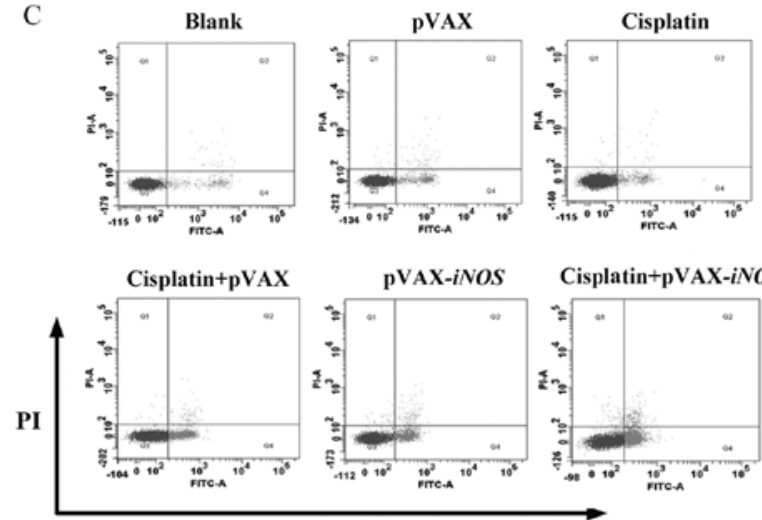

Cisplatin+pVAX-iNOS

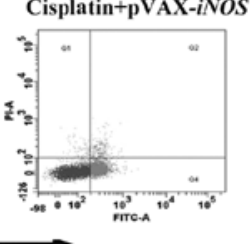

D
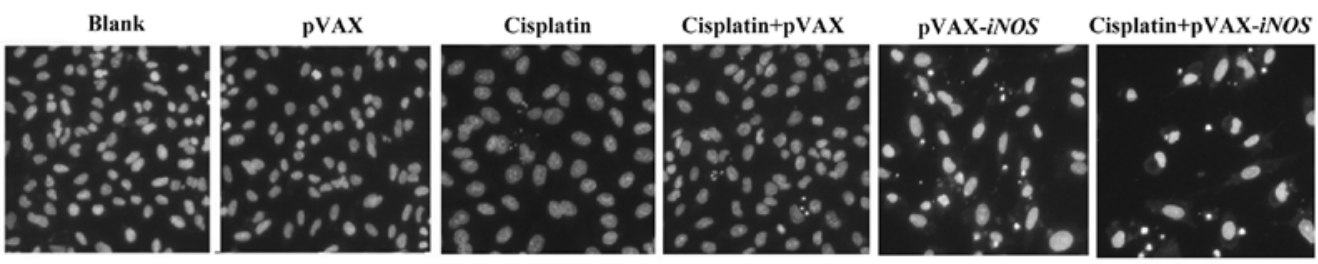

Figure 1. Effect of exogenously enforced high expression of $i N O S$ on cisplatin sensitivity and low-dose cisplatin-mediated cell proliferation inhibition and cell apoptosis promotion in A549 cells. (A) The expression of iNOS protein in A549 cells transfected with LP-pVAX-iNOS was detected. Meanwhile, the sensitivity of A549 cells with the combination treatment of LP-pVAX-iNOS and a series of cisplatin concentrations for $48 \mathrm{~h}$ was analyzed by MTT assay. (B) The proliferation of A549 cells following the combination treatment of LP-pVAX-iNOS and cisplatin at an $\mathrm{IC}_{20}$ dose for $48 \mathrm{~h}$ was analyzed by MTT assay. (C) Cell apoptosis following different treatments was detected by FCM. (D) Apoptosis following different treatments was detected by Hoechst 33258 staining. Columns, the means of 3 individual experiments; bars, SD. ${ }^{*} \mathrm{P}<0.05 ;{ }^{* *} \mathrm{P}<0.01$; Blank, untreated group.

cisplatin, we analyzed the cell viability of the A549 cells following cisplatin treatment alone or the combination treatment with LP-pVAX-iNOS transfection and cisplatin by MTT assay. The $\mathrm{IC}_{50}$ value of cisplatin in the A549 cell line was decreased from $8.80 \pm 1.95 \mu \mathrm{M}$ in the cisplatin alone group to $5.08 \pm 0.73 \mu \mathrm{M}$ in the combination treatment group. Therefore, the results suggest that exogenously enforced high expression of the iNOS gene significantly enhances the sensitivity of A549 cells to cisplatin.

Enhanced proliferation inhibition following the combination treatment of LP-pVAX-iNOS and low-dose cisplatin. To examine whether the exogenously enforced high expression of the $i N O S$ gene enhances cisplatin-mediated cell proliferation inhibition, the $\mathrm{IC}_{20}$ dose level $(3.05 \pm 1.25 \mu \mathrm{M})$ of cisplatin in A549 cells was adopted in the following experiments. The cell viability of A549 cells following different treatments was assessed by MTT assay. There was $>30$ and $15 \%$ of an average decrease in cell viability at $48 \mathrm{~h}$ in the combination treatment group compared with the low-dose cisplatin alone or iNOS gene alone groups $(\mathrm{P}<0.01)$ (Fig. 1B). The results indicated that the exogenously enforced high expression of the $i N O S$ gene significantly enhances the proliferation inhibition of low-dose cisplatin in A549 cells.

Enhanced induction of apoptosis following combination treatment of LP-pVAX-iNOS and low-dose cisplatin. To evaluate whether the enforced high expression of the $i N O S$ gene is implicated in the reactivity of cells to cisplatininduced apoptosis, both Hoechst 33258 and Annexin V-PI staining by flow cytometry were used. Nuclear condensation, cleavage fragments and additional apoptotic bodies appeared in the A549 cells following the combination treatment, while these were rarely noted in the low-dose cisplatin alone or iNOS gene alone treated groups (Fig. 1D). Moreover, the results of Annexin V and PI double staining demonstrated 
A

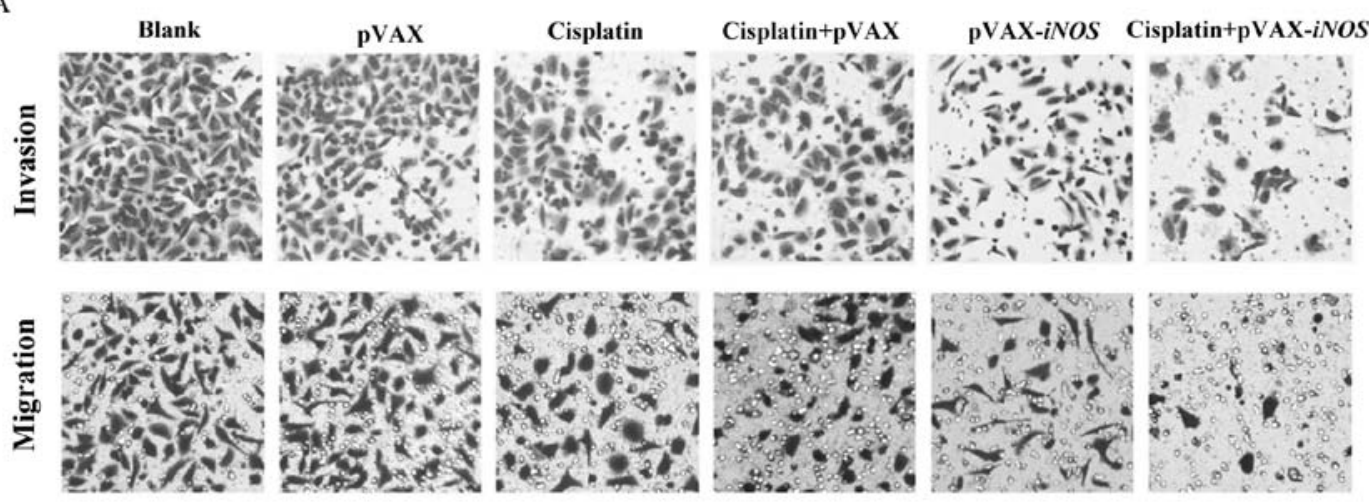

B

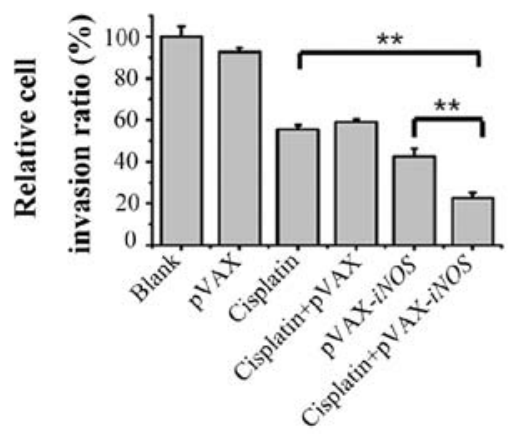

$\mathrm{C}$

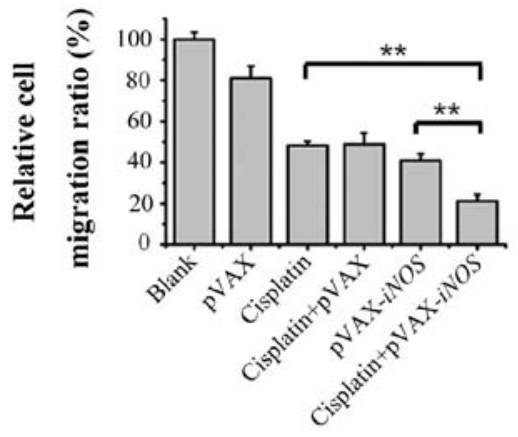

Figure 2. Cell invasion and migration of A549 cells following LP-pVAX-iNOS and/or low-dose cisplatin treatment. (A) Photomicrographs of A549 cells in the lower section of the Boyden cell and Millicell chamber stained with crystal violet. (B) The relative cell invasion ratio of A549 cells under different treatment conditions. (C) The relative cell migration ratio of A549 cells under different treatment conditions. Columns, the means of 3 individual experiments; bars, SD. ${ }^{* *} \mathrm{P}<0.01$. Blank, untreated group.

that the early apoptosis rate in cells following treatment of low-dose cisplatin alone or iNOS gene alone was an average of 8.00 or $20.97 \%$, while significantly enhanced induction of early apoptosis was observed (an average of 31.7\%) in the combination treatment group (Fig. 1C). Taken together, these results implied that the exogenously enforced high expression of the iNOS gene may significantly enhance low-dose cisplatin-mediated cell apoptosis $(\mathrm{P}<0.01)$.

Enhanced inhibition of cell invasion and migration abilities by the combination treatment with LP-pVAX-iNOS and low-dose cisplatin. As distant metastasis is responsible for the failure of lung cancer treatment, assessment of cell invasion and migration ability is significantly important for studying cancer treatment. Moderate inhibition (an average of 44.4 and $57.3 \%$, respectively) of invasion was observed in the A549 cells treated with low-dose cisplatin or the iNOS gene alone, while the combination treatment resulted in significant inhibition (an average of $77.4 \%$ ) of invasion in the A549 cells $(\mathrm{P}<0.05)$ (Fig. 2A and B). Similar results were obtained in the Boyden Millicell assay. As shown in Fig. 2A and C, the changes in the inhibition of migration (an average of 51.8 and $59.1 \%$ respectively) were also considered modest in the A549 cells treated with low-dose cisplatin or the iNOS gene alone, but more significant suppression ( 78.8\%) was observed in the cells following combination treatment with LP-pVAX-iNOS and low-dose cisplatin $(\mathrm{P}<0.05)$. These results indicated that the $i N O S$ gene may significantly enhance the low-dose cisplatin-mediated inhibition of cell invasion and migration in A549 lung cancer cells.

Enhanced tumor growth inhibition and apoptosis induction by the combination treatment with LP-pVAX-iNOS and low-dose cisplatin in vivo. As the enhanced antitumor activity in A549 cells in the combination treatment was observed in vitro, we hypothesized that the same effects may emerge in vivo. To verify this assumption, we established a human A549 lung cancer metastasis mouse model and a subcutaneous tumor xenograft mouse model to evaluate the effects of the $i N O S$ gene on cisplatin-induced tumor regression. In the human A549 lung cancer metastasis mouse model, as shown in Fig. 3A and C, consistent with the in vitro experiment, the combination treatment significantly inhibited tumor growth with an average reduction of $92.37 \%$ compared with an average reduction of 37.25 and $79.67 \%$, respectively, in low-dose cisplatin- or $i N O S$ gene-mediated tumor growth inhibition $(\mathrm{P}<0.01)$. Meanwhile, we evaluated the combination treatment on animal survival in the human A549 lung cancer metastasis mouse models. The combination treatment resulted in a significant and prolonged survival (mean survival time, $200.8 \pm 11.2$ days) compared to the group treated with low-dose cisplatin alone (mean, 133.6 \pm 22.2 days) or treated with LP-pVAX-iNOS alone (mean, 181.2 \pm 8.5 days) (Fig. 3B).

In addition, the A549 cell subcutaneous tumor xenograft mouse model was also established to explore the effects of the $i N O S$ gene on enhancing the cisplatin-induced antitumor 
Table I. Time (days) required for tumors to grow to three times their volume from the day of treatment.

\begin{tabular}{lcccccc}
\hline Treatment & Glucose & pVAX & Cisplatin & Cisplatin + pVAX & pVAX-iNOS & Cisplatin + pVAX-iNOS \\
\hline $\begin{array}{l}\text { Time to reach 3 } \\
\text { times treatment } \\
\text { volume (days) }\end{array}$ & $8.53 \pm 1.04$ & $13.30 \pm 3.76$ & $13.53 \pm 0.94$ & $15.18 \pm 0.88$ & $18.04 \pm 1.72$ & $20.34 \pm 0.88$ \\
\hline
\end{tabular}

A

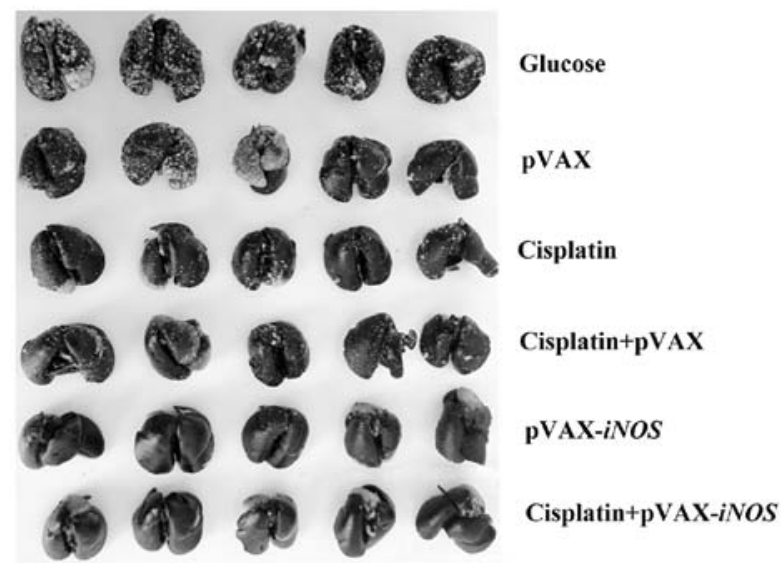

B

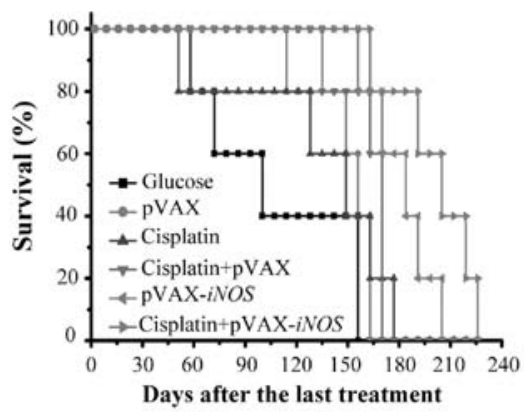

C

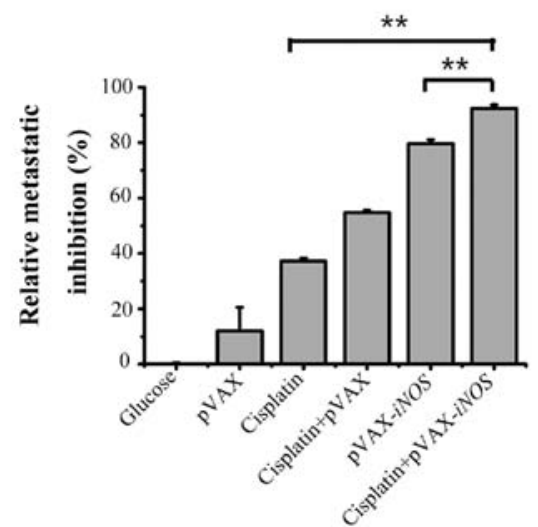

Figure 3. Effect of the combination treatment with LP-pVAX-iNOS and low-dose cisplatin on tumor growth and the life span of mice in the lung metastasis nude mouse model. (A) Metastatic tumors in the lungs of the 6 treatment groups. On the 14th day after the last treatment, mice were anesthetized and their lungs were filled with India ink to examine the number of metastases. (B) Combination treatment prolonged the life span of the mice. Mouse survival curves were plotted according to the Kaplan-Meier method. (C) The percent of metastatic inhibition in each group. Columns, the means of 3 individual experiments; bars, $\mathrm{SD} .{ }^{* *} \mathrm{P}<0.01$.

effects. Combination treatment significantly inhibited tumor growth with an average reduction of $71.99 \%$ compared with a tumor growth reduction of 19.65 and $51.71 \%$, respectively following treatment with a low-dose cisplatin- or the $i N O S$ gene alone $(\mathrm{P}<0.01)$ (Fig. 4A, B and $\mathrm{D})$. The tumors in the glucose-treated groups increased 3 times in volume on day $8.53 \pm 1.04$ after treatment while a single injection of $2 \mathrm{mg} / \mathrm{kg}$ cisplatin or LP-pVAX-iNOS slowed this growth in the tumor volume to $13.53 \pm 0.94$ or $18.04 \pm 1.72$ days, respectively (Table I). Moreover, the combination treatment significantly slowed tumor growth. Tumor growth 3 times the original volume in the combination treatment group was not reached until 20.34 \pm 0.88 days $(\mathrm{P}<0.05)$.

The peeled off subcutaneous tumor tissues were fixed in formalin and further used by TUNEL staining to analyze the apoptosis of tumor tissues. Histological analysis was also applied to detect necrosis/apoptosis in the formalinfixed tumor tissues. As shown in Fig. 4C and E, the tumor tissues in the combination treatment groups displayed more TUNEL-positive nuclei and visible necrotic/apoptotic regions compared to that in the $i N O S$ gene or low-dose cisplatintreated groups. Histological analysis of the various organs demonstrated no significant treatment-related toxicity. Our results further demonstrated that the increased apoptosis in tumor tissues following the combination treatment may be responsible for the enhancement of low-dose cisplatininduced antitumor effects in vivo.

Induction of p-p53 overexpression and suppression of p-mTOR and MMP2 expression by combination treatment with LP-pVAX-iNOS and low-dose cisplatin in vitro. Phosphorylation of p53 plays important roles in both cisplatin- 


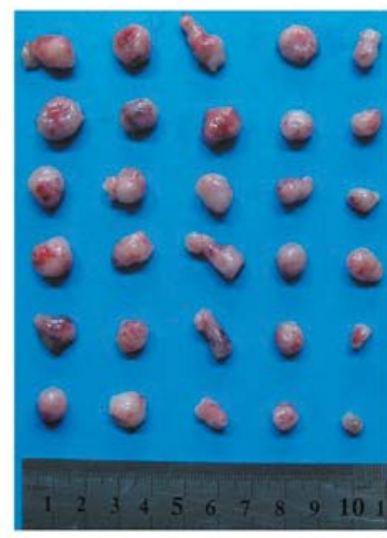

$\mathrm{C}$

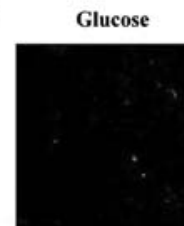

Cisplatin $+p$ VAX

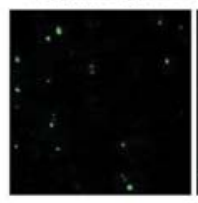

E

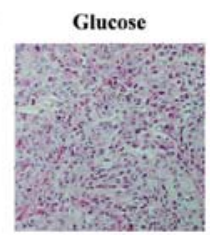

pVAX

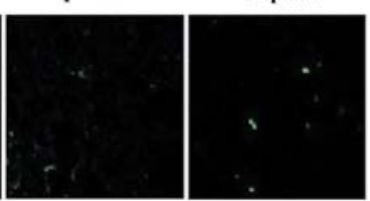

pVAX-iNOS Cisplatin+pVAX-iNOS
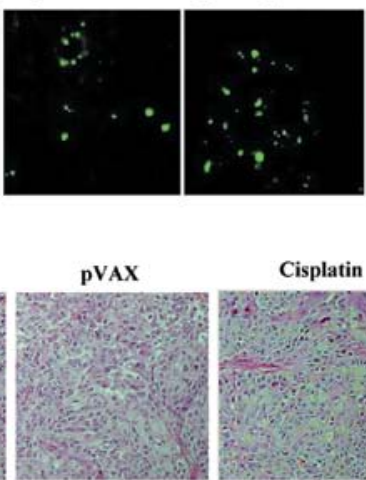

Glucose

pVAX

Cisplatin

pVAX-iNOS
Cisplatin+pVAX

Cisplatin+pVAX-iNOS

B

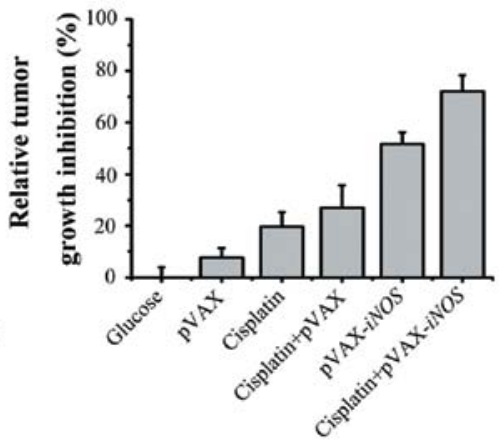

D

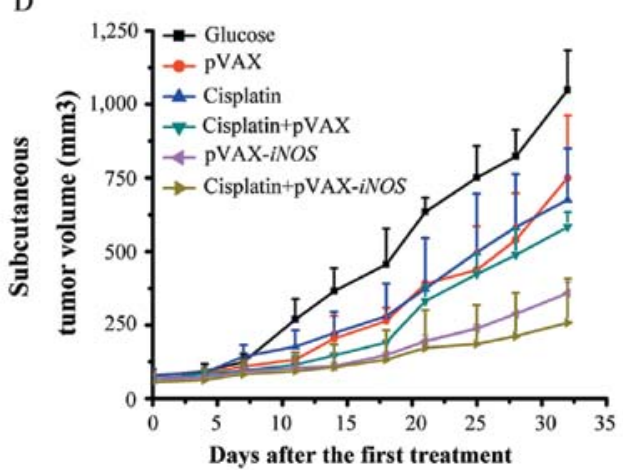

Cisplatin $+p V A X$

pVAX-iNOS Cisplatin+pVAX-iNOS
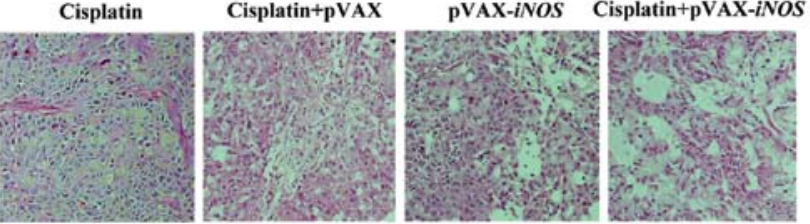

Figure 4. Effect of the combination treatment with LP-pVAX-iNOS and low-dose cisplatin on tumor growth and apoptosis in subcutaneous tumor nude mouse model. (A) Images of the subcutaneous tumor tissues peeled off from the nude mice in 6 groups. (B) The growth inhibition ratio of the subcutaneous tumor in each group. (C) The induction of apoptosis for the subcutaneous tumor tissues. After TUNEL staining of subcutaneous tumor tissues, discrete TUNEL fluorescence signals were observed. (D) The growth curves of each treatment group. Tumor volumes of the subcutaneous lung tumors were recorded at different time periods. (E) Histological analysis of the subcutaneous tumor tissues. The paraffin blocks embedded with subcutaneous mouse tumor tissues were used with $\mathrm{H} \& \mathrm{E}$ staining to analyze the general pathological changes with tumor tissues. Columns, the means of 3 individual experiments; bars, SD. ${ }^{* *} \mathrm{P}<0.01$.

and NO-induced cell apoptosis $(4,35,36)$. To evaluate whether phosphorylation of p53 was implied in the enhanced effects of the combination treatment, we detected the expression levels of p-p53 protein in the different treatment groups by western blotting. The expression of p-p53 protein was increased in cells treated with the $i N O S$ gene or low-dose cisplatin alone, but a dramatic upregulated level of p-p53 protein was detected in cells following the combination treatment (Fig. 5).

mTOR is vital in mediating cisplatin sensitivity (37-39). However, the relationship of mTOR and NO in antitumor processes has not been reported. To examine whether a correlation exists between mTOR and NO, which may participate in the reactivity of cells to low-dose cisplatin after LP-pVAX-iNOS treatment, we analyzed the expression of phosphorylated mTOR protein in the differently treated cells by western blotting. Only a slight downregulation of p-mTOR was detected in cells treated with the iNOS gene or low-dose cisplatin alone, but a dramatic downregulation of p-mTOR was observed in cells after the combination treatment (Fig. 5).

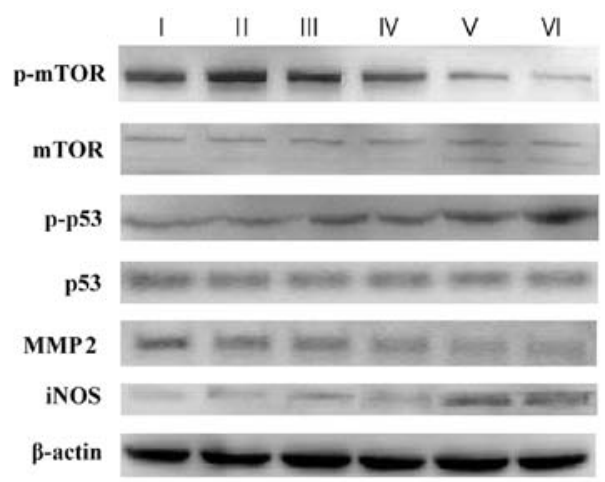

Figure 5. The in vitro expression of related proteins in the various treated cell groups by western blotting. After treatment with LP-pVAX-iNOS and/ or cisplatin, A549 cells were collected and lysated. The total cellular lysates were analyzed by western blotting with special antibodies including, iNOS, p-mTOR, mTOR, p-p53, p53, MMP2 and $\beta$-actin (an internal control). Lane I, untreated group; lane II, pVAX treated group; lane III, cisplatin-treated group; lane IV, cisplatin plus pVAX-treated group; lane V, pVAX-iNOS-treated group; lane VI, cisplatin plus pVAX-iNOS-treated group. 
MMP2 is also an important kinase in the process of cell invasion. To explore whether MMP2 protein was involved in the enhanced effects of the combination treatment, we also detected the expression levels of the MMP2 protein in the different treatment groups by western blotting. The expression level of the MMP2 protein in A549 cells after the combination treatment was also significantly downregulated compared with that in cells treated with the iNOS gene or low-dose cisplatin alone (Fig. 5).

\section{Discussion}

Cisplatin is one of the first-line chemotherapeutic drugs used in the clinical treatment of lung cancer patients. However, the side effects and drug resistance restrict its wide use. The important task of cancer therapy is to seek a suitable method to enhance the sensitivity of existing chemotherapeutic agents (25). Thus, refinement for enhancing the sensitivity of cisplatin is highly required.

As previous studies reported, high NO levels generated from $i N O S$ gene transfer, cytokine stimulation or NO donor may have antitumor effects and even enhance the cytotoxicity of the chemotherapeutic drug cisplatin in RIF-1 tumors, ovarian cancer, leukemia, prostate or colon cancer cells $(18,26,27)$. Among all the methods used for high concentrations of NO production, iNOS gene transfer may be accepted as a superior way due to its marked 'bystander' effect and safety $(11,12,14)$. Only one article previously demonstrated that $i N O S$ gene transfer treatment may increase the cytotoxicity and cause a delay in the growth of cisplatintreated RIF-1 tumors, prostate and colon cancer cells (18). However, whether this combined efficacy is observed in other types of tumors remains unknown. Moreover, whether the combination treatment affects the invasion and metastasis of cancer cells, which is important in cancer treatment, remains unclear.

Consistent with the above-mentioned study, we also showed that the $\mathrm{IC}_{50}$ of cisplatin was reduced in A549 cells after transfection with the $i N O S$ gene. The combination treatment significantly inhibited the growth of subcutaneous tumors. Most importantly, we further aimed to clarify that $i N O S$ gene therapy may significantly enhance the antitumor effects of cisplatin through the promotion of cell apoptosis, as well as effective inhibition of proliferation, invasion and migration abilities in vitro and in vivo. In order to simulate the metastatic characteristics of lung cancer cells in vivo, an A549 lung metastatic tumor- bearing mouse model was established. The combination treatment significantly suppressed the formation of lung metastases via systemic administration of tail vein injection of LP-pVAX or LP-pVAX-iNOS (20 $\mu \mathrm{g}$ DNA/mouse) and/or i.p. injection of low-dose cisplatin $(2 \mathrm{mg} / \mathrm{kg} / \mathrm{mouse})$ and dramatically prolonged the life spans of tumor-bearing mice with no significant organ-related toxicity. Systemic administration of the LP-DNA complex is a novel drug delivery method that has been shown to deliver genes effectively to the lungs when administered intravenously (40). More importantly, systemic administration is more suitable for lung cancer patients in clinical treatment procedure. To the best of our knowledge, this is the first report demonstrating that exogenously enforced high expression of the $i N O S$ gene by cationic liposome (LP)-mediated $i N O S$ gene transfer in lung cancer A549 cells increases the cisplatin sensitivity and significantly enhances the antitumor effects of cisplatin in human lung cancer A549 cells both in vitro and in vivo. The iNOS gene significantly enhanced the cisplatin-mediated inhibition of lung cancer cell migration and invasion. Tumor metastasis is responsible for approximately $90 \%$ of lung cancer-related death (41). The combination treatment with $i N O S$ gene therapy and low-dose cisplatin would be a novel and potential strategy for lung cancer treatment (24).

The mechanisms of cisplatin- or NO-based antitumor activity have been basically reported (35-37,42,43). Studies suggest that both p53 and mTOR signaling pathways are important in cisplatin-mediated antitumor activity $(35,37,42)$. Meanwhile, the antitumor effects of NO generated by iNOS gene transfer were reported to be at least partly dependent on the phosphorylation of p53 and MMP2 $(36,44)$. However, the associated mechanisms of the combined effects of iNOS gene therapy and cisplatin in human cancers have not been reported. Thus, to reveal the molecular mechanisms of iNOS involved in enhancing low-dose cisplatin-mediated antitumor activities, we further detected the expression levels of these key proteins in NO-mediated and/or cisplatin-mediated signaling pathways by western blotting.

p53 is an important marker in the process of cell apoptosis (4), which is the main mode of cisplatin-induced cell death. Cisplatin sensitivity is closely related with the presence of the pro-apoptotic protein p53 (35). Meanwhile, studies have demonstrated that a high level of NO resulting from the high expression of the $i N O S$ gene may promote cell apoptosis in melanoma, renal cell cancer and their adjacent cells $(7,44)$. Cook et al $(36)$ reported that the pro-apoptotic mechanisms of NO generated by $i N O S$ gene transfer involved in the death signaling pathway were at least partly dependent on the phosphorylation of p53. When p53 was knocked out, the combination treatment of $i N O S$ gene therapy and radiotherapy reduced the incidence of tumor cell apoptosis and antitumor effects in colon cancer cells. Consistent with previous studies, we also observed that iNOS gene therapy may significantly enhance the low-dose cisplatin-mediated apoptosis of human lung cancer A549 cells. Similarly, the dramatic upregulation of p-p53 protein expression was observed in the combination treatment group, while a slight upregulation of $\mathrm{p}$-p53 protein expression was noted in the $i N O S$ gene or cisplatin alone treatment group. The results indicated that $i N O S$ gene therapy enhancing the antitumor effects of low-dose cisplatin in lung cancer may be partly related to the upregulated expression of p-p53 protein.

mTOR is a serine/threonine protein kinase, which plays an important role in the regulation of cell functions including cell proliferation, cell cycle, biosynthesis and cell migration (42). Meanwhile, mTOR protein is one of the widely studied kinases involved in the main signaling pathway of cisplatin. A high degree of intracellular phosphorylation of mTOR was often noted in several cisplatin-resistant cancer cells, including NLCLC cells $(37,38)$, which indicates that inhibition of mTOR activity may enhance cancer cell sensitivity to cisplatin $(37,39)$. Currently, the relationship between NO-mediated antitumor effects and p-mTOR protein expres- 
sion has not been reported. To the best of our knowledge, our study first discovered that the expression of p-mTOR protein was significantly diminished in the combination treatment group compared with the $i N O S$ gene or cisplatin alone-treated group. The results suggested that the downregulated expression of p-mTOR protein may be another probable reason for iNOS enhancing low-dose cisplatin-mediated inhibition of metastasis and invasion in lung cancer.

Metastasis is responsible for the poor effect of clinical treatment in lung cancer. Matrix metalloproteinases (MMPs) play a key role in the process of tumor metastasis (45). Karam et al (46) reported that cisplatin may inhibit the invasion and migration of human ovarian cancer cells by downregulating the expression of MMP2, TIMP1 and TIMP2. Another study also discovered that an increased amount of cisplatin resulted in a time- and dose-dependent decreased level of the MMP2 protein in transformed rat thyroid cancer cells (47). Meanwhile, iNOS gene activity was inversely related to the metastasis of tumor cells $(4,7)$. NO may affect the invasion of mouse mammary adenocarcinoma through breaking the balance between MMP2 and its inhibitors, including tissue inhibitor of metalloproteinase (TIMP2 and TIMP3) (43). When the high concentration of NO occurred, the expression levels of MMPs gradually decreased (48). In our study, we discovered that $i N O S$ gene therapy significantly reduced the low-dose cisplatin-mediated invasion and migration capacity in lung cancer A549 cells and the expression levels of the MMP2 protein were downregulated in the combination treatment group compared with the $i N O S$ gene or cisplatin alone treated groups. The results indicated that $i N O S$ gene therapy enhancing the antitumor effects of low-dose cisplatin in lung cancer may occur through the downregulation of MMP2 protein expression.

In conclusion, our study confirmed that the combination treatment with cationic LP-mediated iNOS gene therapy and low-dose cisplatin may significantly enhance cisplatinmediated cell apoptosis and inhibition of cell proliferation, metastasis and invasion in human lung adenocarcinoma A549 cells in vitro and in vivo. The enhanced antitumor effects of low-dose cisplatin by $i N O S$ gene therapy in lung adenocarcinoma is associated with the upregulation of p-p53 expression and the downregulation of MMP2 and p-mTOR protein expression. Therefore, the combination treatment of iNOS gene therapy and cisplatin is an effective strategy for the treatment of lung cancer.

\section{Acknowledgements}

This study was partly supported by the National Natural Science Foundation of China (no. 81071863). The authors thank Xiang Chen and Qiaorong Huang (State Key Laboratory of Biotherapy and Cancer Center, West China Hospital, Sichuan University) for their technical assistance.

\section{References}

1. Jemal A, Siegel R, Ward E, Murray T, Xu J, Smigal C and Thun MJ: Cancer statistics, 2006. CA Cancer J Clin 56: 106-130, 2006.

2. Jamieson ER and Lippard SJ: Structure, recognition and processing of cisplatin-DNA adducts. Chem Rev 99: 2467-2498, 1999.
3. Molina JR, Adjei AA and Jett JR: Advances in chemotherapy of non-small cell lung cancer. Chest 130: 1211-1219, 2006.

4. Xie K and Fidler IJ: Therapy of cancer metastasis by activation of the inducible nitric oxide synthase. Cancer Metastasis Rev 17: 55-75, 1998.

5. Xie QW, Cho HJ, Calaycay J, Mumford RA, Swiderek KM, Lee TD, Ding A Troso T and Nathan C: Cloning and characterization of inducible nitric oxide synthase from mouse macrophages. Science 256: 225-228, 1992.

6. Heller A: Apoptosis-inducing high (.)NO concentrations are not sustained either in nascent or in developed cancers. ChemMedChem 3: 1493-1499, 2008.

7. Lala PK and Chakraborty C: Role of nitric oxide in carcinogenesis and tumour progression. Lancet Oncol 3: 149-156, 2001.

8. Fujimoto H, Ando Y, Yamashita T, Terazaki H, Tanaka Y, Sasaki J, Matsumoto M, Suga M and Ando M: Nitric oxide synthase activity in human lung cancer. Jpn J Cancer Res 88: 1190-1198, 1997.

9. Puhakka A, Kinnula V, Näpänkangas U, Säily M, Koistinen P, Pääkkö P and Soini Y: High expression of nitric oxide synthases is a favorable prognostic sign in non-small cell lung carcinoma. APMIS 111: 1137-1146, 2003.

10. Fitzpatrick B, Mehibel M, Cowen RL and Stratford IJ: iNOS as a therapeutic target for treatment of human tumors. Nitric Oxide 19: $217-224,2008$

11. Khare PD, Shao-Xi L, Kuroki M, Hirose Y, Arakawa F, Nakamura K, Tomita Y and Kuroki M: Specifically targeted killing of carcinoembryonic antigen (CEA)-expressing cells by a retroviral vector displaying single-chain variable fragmented antibody to CEA and carrying the gene for inducible nitric oxide synthase. Cancer Res 61: 370-375, 2001.

12. Xu W, Liu L and Charles IG: Microencapsulated iNOSexpressing cells cause tumor suppression in mice. FASEB J 16: 213-215, 2002.

13. Soler MN, Bobé P, Benihoud K, Lemaire G, Roos BA and Lausson S: Gene therapy of rat medullary thyroid cancer by naked nitric oxide synthase II DNA injection. J Gene Med 2: 344-352, 2000.

14. Lumniczky K and Safrany G: Cancer gene therapy: combination with radiation therapy and the role of bystander cell killing in the anti-tumor effect. Pathol Oncol Res 12: 118-124, 2006.

15. Siegel R, Ward E, Brawley O and Jemal A: Cancer statistics, 2011: the impact of eliminating socioeconomic and racial disparities on premature cancer deaths. CA Cancer J Clin 61: 212-236, 2011.

16. Ropponen KM, Kellokoski JK, Lipponen PK, Eskelinen MJ, Alanne L, Alhava EM and Kosma VM: Expression of inducible nitric oxide synthase in colorectal cancer and its association with prognosis. Scand J Gastroenterol 35: 1204-1211, 2000.

17. Raspollini MR, Amunni G, Villanucci A, Boddi V, Baroni G, Taddei A and Taddei GL: Expression of inducible nitric oxide synthase and cyclooxygenase-2 in ovarian cancer: correlation with clinical outcome. Gynecol Oncol 92: 806-812, 2004.

18. Adams C, McCarthy HO, Coulter JA, Worthington J, Murphy C, Robson T and Hirst DG: Nitric oxide synthase gene therapy enhances the toxicity of cisplatin in cancer cells. J Gene Med 11: $160-168,2009$

19. Worthington J, McCarthy HO, Barrett E, Adams C, Robson T and Hirst DG: Use of the radiation-inducible WAF1 promoter to drive iNOS gene therapy as a novel anti-cancer treatment. J Gene Med 6: 673-680, 2004.

20. Worthington J, Robson T, O'Keeffe M and Hirst DG: Tumour cell radiosensitization using constitutive (CMV) and radiation inducible (WAF1) promoters to drive the iNOS gene: a novel suicide gene therapy. Gene Ther 9: 263-269, 2002.

21. McCarthy HO, Worthington J, Barrett E, Cosimo E, Boyd M, Mairs RJ, Ward C, McKeown SR, Hirst DG and Robson T: p21 (WAF1)-mediated transcriptional targeting of inducible nitric oxide synthase gene therapy sensitizes tumours to fractionated radiotherapy. Gene Ther 14: 246-255, 2007.

22. Coulter JA, McCarthy HO, Worthington J, Robson T, Scott S and Hirst DG: The radiation-inducible pE9 promoter driving inducible nitric oxide synthase radiosensitizes hypoxic tumour cells to radiation. Gene Ther 15: 495-503, 2008.

23. Wang Z, Cook T, Alber S, Liu K, Kovesdi I, Watkins SK, Vodovotz Y, Billiar TR and Blumberg D: Adenoviral gene transfer of the human inducible nitric oxide synthase gene enhances the radiation response of human colorectal cancer associated with alterations in tumor vascularity. Cancer Res 64: 1386-1395, 2004 
24. Evig CB, Kelley EE, Weydert CJ, Chu Y, Buettner GR and Burns CP: Endogenous production and exogenous exposure to nitric oxide augment doxorubicin cytotoxicity for breast cancer cells but not cardiac myoblasts. Nitric Oxide 10: 119-129, 2004.

25. Wink DA, Cook JA, Christodoulou D, Krishna MC, Pacelli R, Kim S, DeGraff W, Gamson J, Vodovotz Y, Russo A and Mitchell JB: Nitric oxide and some nitric oxide donor compounds enhance the cytotoxicity of cisplatin. Nitric Oxide 1: 88-94, 1997.

26. Son KK and Hall KJ: Nitric oxide-mediated tumor cell killing of cisplatin-based interferon-gamma gene therapy in murine ovarian carcinoma. Cancer Gene Ther 7: 1324-1328, 2000.

27. Konovalova NP, Goncharova SA, Volkova LM, Rajewskaya TA, Eremenko L and Korolev AM: Nitric oxide donor increases the efficiency of cytostatic therapy and retards the development of drug resistance. Nitric Oxide 8: 59-64, 2003.

28. Chen JH, Lin HH, Chiang TA, Hsu JD, Ho HH, Lee YC and Wang CJ: Gaseous nitrogen oxide promotes human lung cancer cell line A549 migration, invasion, and metastasis via iNOSmediated MMP-2 production. Toxicol Sci 106: 364-375, 2008.

29. Kisley LR, Barrett BS, Bauer AK, Dwyer-Nield LD, Barthel B Meyer AM, Thompson DC and Malkinson AM: Genetic ablation of inducible nitric oxide synthase decreases mouse lung tumorigenesis. Cancer Res 62: 6850-6856, 2002.

30. Chen X, Wang X, Wang Y, Yang L, Hu J, Xiao W, Fu A, Cai L, Li X, Ye X, Liu Y, et al: Improved tumor-targeting drug delivery and therapeutic efficacy by cationic liposome modified with truncated bFGF peptide. J Control Release 145: 17-25, 2010.

31. Shamimi-Noori S, Yeow W-S, Ziauddin MF, Xin H, Tran TL, Xie J, Loehfelm A, Patel P, Yang J, Schrump DS, et al: Cisplatin enhances the antitumor effect of tumor necrosis factor-related apoptosis-inducing ligand gene therapy via recruitment of the mitochondria-dependent death signaling pathway. Cancer Gene Ther 15: 356-370, 2008.

32. Ito I, Ji L, Tanaka F, Saito Y, Gopalan B, Branch CD, Xu K, Atkinson EN, Bekele BN, Stephens LC, et al: Liposomal vector mediated delivery of the $3 p$ FUS1 gene demonstrates potent antitumor activity against human lung cancer in vivo. Cancer Gene Ther 11: 733-739, 2004.

33. Wei YQ, Wang QR, Zhao X, Yang L, Tian L, Lu Y, Kang B, Lu CJ, Huang MJ, Lou YY, et al: Immunotherapy of tumors with xenogeneic endothelial cells as a vaccine. Nat Med 6: 1160-1166, 2000 .

34. Fraser M, Chan SL, Chan SS, Fiscus RR and Tsang BK: Regulation of p53 and suppression of apoptosis by the soluble guanylyl cyclase/cGMP pathway in human ovarian cancer cells. Oncogene 25: 2203-2212, 2006.

35. Nguyen DM, Spitz FR, Yen N, Cristiano RJ and Roth JA: Gene therapy for lung cancer: enhancement of tumor suppression by a combination of sequential systemic cisplatin and adenovirusmediated p53 gene transfer. J Thorac Cardiovasc Surg 112: 1372-1377, 1996.
36. Cook T, Wang Z, Alber S, Liu K, Watkins SC, Vodovotz Y, Billiar TR and Blumberg D: Nitric oxide and ionizing radiation synergistically promote apoptosis and growth inhibition of cancer by activating p53. Cancer Res 64: 8015-8021, 2004.

37. Wangpaichitr M, Wu C, You M, Kuo MT, Feun L, Lampidis T and Savaraj N: Inhibition of mTOR restores cisplatin sensitivity through down-regulation of growth and anti-apoptotic proteins. Eur J Pharmacol 591: 124-127, 2008.

38. Stewart DJ: Mechanisms of resistance to cisplatin and carboplatin. Crit Rev Oncol Hematol 63: 12-31, 2007.

39. Mabuchi S, Kawase C, Altomare DA, Morishige K, Sawada K, Hayashi M, Tsujimoto M, Yamoto M, Klein-Szanto AJ, Schilder RJ, et al: $\mathrm{mTOR}$ is a promising therapeutic target both in cisplatin-sensitive and cisplatin-resistant clear cell carcinoma of the ovary. Clin Cancer Res 15: 5404-5413, 2009.

40. Templeton NS, Lasic DD, Frederik PM, Strey HH, Roberts DD and Pavlakis GN: Improved DNA:liposome complexes for increased systemic delivery and gene expression. Nat Biotechnol 15: 647-652, 1997.

41. Keshamouni V, Arenberg D and Kalemkeriam G (eds): Lung Cancer Metastasis: Novel Biological Mechanisms and Impact on Clinical Practice. Springer, New York, pp1-395, 2009.

42. Guertin DA and Sabatini DM: Defining the role of mTOR in cancer. Cancer Cell 12: 9-23, 2007.

43. Orucevic A, Bechberger J, Green AM, Shapiro RA, Billiar TR and Lala PK: Nitric-oxide production by murine mammary adenocarcinoma cells promotes tumor-cell invasiveness. Int J Cancer 81: 889-896, 1999.

44. Obara $\mathrm{H}$ and Harasawa $\mathrm{R}$ : Nitric oxide causes anoikis through attenuation of E-cadherin and activation of caspase-3 in human gastric carcinoma AZ-521 cells infected with Mycoplasma hyorhinis. J Vet Med Sci 72: 869-874, 2010.

45. Bjorklund $\mathrm{M}$ and Koivunen E: Gelatinase-mediated migration and invasion of cancer cells. Biochim Biophys Acta 1755: 37-69, 2005.

46. Karam AK, Santiskulvong C, Fekete M, Zabih S, Eng C and Dorigo O: Cisplatin and PI3kinase inhibition decrease invasion and migration of human ovarian carcinoma cells and regulate matrix-metalloproteinase expression. Cytoskeleton (Hoboken) 67: 535-544, 2010.

47. Urso L, Muscella A, Calabriso N, Vetrugno C, Jiménez E, Montiel $\mathrm{M}$ and Marsigliante S: Effects of cisplatin on matrix metalloproteinase-2 in transformed thyroid cells. Biochem Pharmacol 79: 810-816, 2010.

48. Ridnour LA, Thomas DD, Switzer C, Flores-Santana W, Isenberg JS, Ambs S, Roberts DD and Wink DA: Molecular mechanisms for discrete nitric oxide levels in cancer. Nitric Oxide 19: 73-76, 2008. 